Supporting Information

\title{
Modification of a Polymer Surface by Partial Swelling Using Non-solvents
}

Hidenobu Taneda, ${ }^{\dagger}$ Norifumi. L. Yamada,${ }^{\ddagger}$ Fumiya Nemoto $₫, \uparrow$ Yasuhisa Minagawa, ${ }^{\#}$ Hisao Matsuno, ${ }^{*}, \dagger, \S$ and Keiji Tanaka*,†,§

$\dagger$ Department of Applied Chemistry, Kyushu University, 744 Motooka, Nishi-ku, Fukuoka 819-0395, Japan.

Neutron Science Laboratory, High Energy Accelerator Research Organization, 203-1 Shirakata, Tokai, Naka-gun, Ibaraki 319-1106, Japan.

\#Sumitomo Rubber Industries, Ltd., 2-1-1 Tsutsui-cho, Chuo-ku, Kobe 651-0071, Japan.

$\S$ Center for Polymer Interface and Molecular Adhesion Science, Kyushu University, 744 Motooka, Nishi-ku, Fukuoka 819-0395, Japan.

†Present address: Department of Materials Science and Engineering, National Defense Academy, 1-1020 Hashirimizu, Yokosuka, Kanagawa 239-8686, Japan

*To whom correspondence should be addressed

FAX: $+81-92-802-2880 \quad$ TEL: $+81-92-802-2878$

E-mail: h-matsuno@cstf.kyushu-u.ac.jp \& k-tanaka@cstf.kyushu-u.ac.jp

KEYWORDS: surface, interface, polymer films, non-solvents, anti-biofouling properties 


\section{SURFACE AND INTERFACE CHARACTERIZATIONS}

1.1. Hansen Solubility Parameter (HSP) Distance $\left(\boldsymbol{R}_{\mathrm{a}}\right)$. Table S1 summarizes HSPs used for the calculation of $R_{\mathrm{a}}$ between polystyrene (PS) and each alcohol. ${ }^{\mathrm{S} 1} \delta_{\mathrm{d}}, \delta_{\mathrm{p}}$, and $\delta_{\mathrm{h}}$ denote dispersion, polar, and hydrogen bonding terms, respectively.

Table S1. HSPs used for calculation of $R_{\mathrm{a}}$ between PS and each alcohol. ${ }^{\mathrm{S} 1}$

\begin{tabular}{ccccc}
\hline \multirow{2}{*}{ materials } & \multicolumn{3}{c}{$\mathrm{HSP} /(\mathrm{MPa})^{0.5}$} \\
\cline { 2 - 2 } PS & $\delta_{\mathrm{d}} /(\mathrm{MPa})^{0.5} /(\mathrm{MPa})^{0.5}$ & & $\delta_{\mathrm{h}} /(\mathrm{MPa})^{0.5}$ \\
\hline methanol & 18.5 & & 4.5 & 2.9 \\
1-propanol & 15.1 & & 12.3 & 22.3 \\
1-butanol & 16.0 & & 6.8 & 17.4 \\
1-hexanol & 16.0 & & 5.7 & 15.8 \\
1-octanol & 15.9 & & 5.8 & 12.5 \\
\hline
\end{tabular}

The $R_{\mathrm{a}}$ value between component 1 and component 2 was calculated according to the following equation,

$$
R_{a}=\left[4\left(\delta_{\mathrm{d} 1}-\delta_{\mathrm{d} 2}\right)^{2}+\left(\delta_{\mathrm{p} 1}-\delta_{\mathrm{p} 2}\right)^{2}+\left(\delta_{\mathrm{p} 1}-\delta_{\mathrm{p} 2}\right)^{2}\right]^{0.5}
$$

where subscript numbers mean each component.

1.2. Atomic Force Microscopy (AFM). Panels $(a-d)$ of Figure S1 represent AFM (a, c) height and (b, d) phase histograms for ( $a, b)$ intact PS and (c, d) poly(2-methoxyethyl acrylate) (PMEA)-treated PS films, respectively. Unlike the PS film case showing simple distribution, the histogram for the PMEAtreated PS showed multiple distributions indicating the overlayer of PMEA was formed on the PS surface.
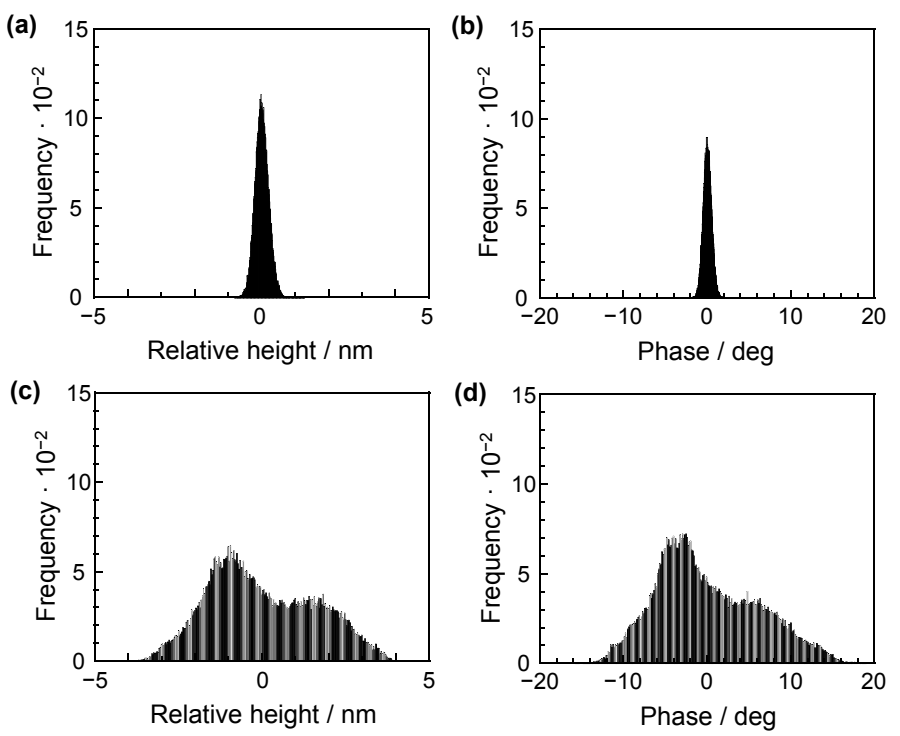
Figure S1. AFM (a, c) height and $(b, d)$ phase histograms for $(a, b)$ intact PS and (c, d) PMEA-PS films, respectively.

1.3. Neutron Reflectivity (NR) Analysis. Table S2 summarizes parameters used to fit NR data for films of deuterated PS (dPS) and PMEA-treated dPS (PMEA-dPS) acquired in air shown in panel (a) of Figure 3. $(b / V)_{\mathrm{i}}, t_{\mathrm{i}}$, and $\sigma_{\mathrm{i}}$ represent scattering length density, thickness, and Gaussian roughness for layer $\mathrm{i}$, respectively. $\xi$ is a decay length when describing the PMEA density profile at the PS interface by a single exponential function. Table S3 summarizes parameters used to fit NR data for a PMEA-dPS film acquired in $\mathrm{D}_{2} \mathrm{O}$ or $\mathrm{H}_{2} \mathrm{O}$ shown in Figure 5(a).

Table S2. Parameters to fit NR data for dPS and PMEA-dPS films acquired in air shown in Figure 3(a).

\begin{tabular}{|c|c|c|c|c|c|c|c|c|c|c|c|c|}
\hline \multirow{2}{*}{ Film } & \multicolumn{4}{|c|}{$(b / V)_{\mathrm{i}} \cdot 10^{-4} / \mathrm{nm}$} & \multicolumn{5}{|c|}{$t_{\mathrm{i}} / \mathrm{nm}$} & \multicolumn{2}{|c|}{$\sigma_{\mathrm{i}} / \mathrm{nm}$} & \multirow{2}{*}{$\xi / \mathrm{nm}$} \\
\hline & $(b / V)_{1}$ & $(b / V)_{\mathrm{I}}$ & $(b / V)_{\text {II }}$ & $(b / V)_{\text {III }}$ & $t_{1}$ & $t_{2}$ & $t_{\mathrm{I}}$ & $t_{\mathrm{II}}$ & $t_{\text {III }}$ & $\sigma_{0}$ & $\sigma_{1}$ & \\
\hline $\mathrm{dPS}$ & 6.22 & - & - & - & 55.8 & - & - & - & - & 0.3 & 0.3 & - \\
\hline PMEA-dPS & 6.22 & 1.15 & 0.89 & 0.42 & 57.3 & 4.0 & 0.7 & 1.2 & 2.1 & 0.3 & - & 1.2 \\
\hline
\end{tabular}

Table S3. Parameters to fit NR data for a PMEA-dPS film acquired in $\mathrm{D}_{2} \mathrm{O}$ or $\mathrm{H}_{2} \mathrm{O}$ shown in Figure 5(a).

\begin{tabular}{|c|c|c|c|c|c|c|c|c|c|c|c|c|}
\hline \multirow{2}{*}{ medium } & \multicolumn{4}{|c|}{$(b / V)_{\mathrm{i}} \cdot 10^{-4} / \mathrm{nm}$} & \multicolumn{5}{|c|}{$t_{\mathrm{i}} / \mathrm{nm}$} & \multicolumn{2}{|c|}{$\sigma_{\mathrm{i}} / \mathrm{nm}$} & \multirow{2}{*}{$\xi / \mathrm{nm}$} \\
\hline & $(b / V)_{1}$ & $(b / V)_{\mathrm{I}}$ & $(b / V)_{\mathrm{II}}$ & $(b / V)_{\text {III }}$ & $t_{1}$ & $t_{2}$ & $t_{\mathrm{I}}$ & $t_{\mathrm{II}}$ & $t_{\text {III }}$ & $\sigma_{0}$ & $\sigma_{1}$ & \\
\hline $\mathrm{D}_{2} \mathrm{O}$ & 6.22 & 1.97 & 3.02 & 5.07 & 57.4 & 5.9 & 0.7 & 1.7 & 3.5 & 0.3 & - & 1.2 \\
\hline $\mathrm{H}_{2} \mathrm{O}$ & 6.22 & 0.88 & 0.54 & -0.13 & 57.4 & 5.9 & 0.7 & 1.7 & 3.5 & 0.3 & - & 1.2 \\
\hline
\end{tabular}

\section{PLATELET ADHESION TEST}

Panels $(a, b)$ of Figure S2 show scanning electron microscopic (SEM) images for platelets adhered onto PS films on which PMEA with different number-average of molecular weights $\left(M_{\mathrm{n}} \mathrm{s}\right)$ of (a) $22 \mathrm{k}$ and (b) 236k were incorporated, respectively. Panel (c) of Figure S2 shows the number of platelets $\left(N_{\text {PLT }}\right)$ and its breakdown classified into three levels on the basis of the degree of activation of platelets described in the main text.
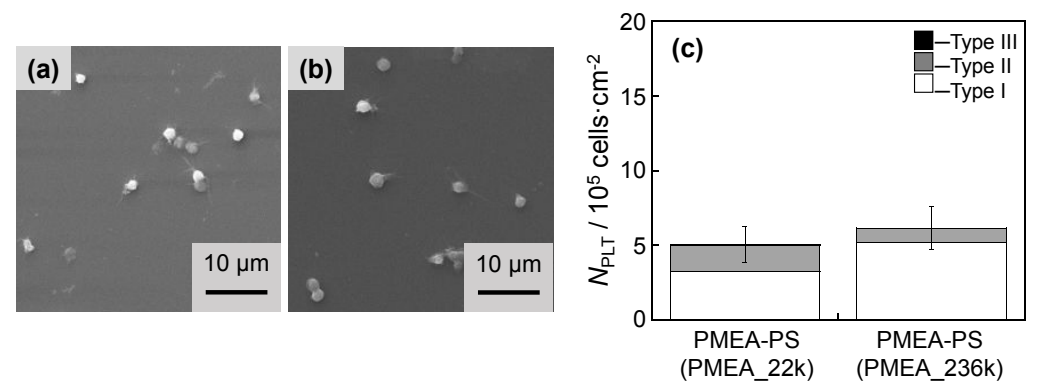
Figure S2. (a, b) SEM images for platelets adhered on PMEA-PS films; $M_{\mathrm{n}} \mathrm{S}$ of PMEAs were (a) $22 \mathrm{k}$ and (b) 236k, respectively. (c) The $N_{\text {PLT }}$ value for each sample classified into three activation degrees of platelets on the basis of the morphology.

\section{REFERENCE}

S1. Hansen C. M. Hansen Solubility Parameters: A User's Handbook, 2nd ed.; CRC Press: Boca Raton, FL; 2007. 